\title{
Controversial Topics The Mechanism of Insulin Action
}

\author{
The Interaction of Insulin with its Receptor: \\ Cross-linking via Insulin Association as the Source of Receptor Clustering
}

\author{
P.D.Jeffrey \\ Department of Physical Biochemistry, John Curtin School of Medical Research, The Australian National University, Canberra, Australia
}

\begin{abstract}
Summary. The extensive association of mammalian insulins in solution and the aggregation of insulin receptors in cell membranes are well documented. The hypothesis advanced here is that a direct connection exists between these observations. It is postulated that, after binding to its receptor, an insulin monomer can interact with another similarly bonded hormone-receptor complex through those groups on the insulin monomer faces utilized for dimer-dimer contacts in the crystal and in solution. Regarded thus, the insulin molecules are effectively bivalent as required for the formation of cross-links
\end{abstract}

between receptors, with the accompanying enhancement of biological activity. A number of properties of native insulins from different animals, and of modified insulins, are considered in the light of this suggestion. It is shown to have considerable power in reconciling a diversity of such observations and to provide a plausible model for the experimentally observed receptor clustering phenomenon.

Key words: Insulin, insulin activity, insulin association, crosslinking, receptor clustering.
The primary event in the biological activity of insulin is the binding of an insulin monomer to a specific cell membrane receptor site [1]. There is convincing evidence, both from experiments involving visualization of fluorescently-labelled insulin derivatives. [2], and from those utilizing other multivalent molecules giving rise to insulin-like effects [3], that the initial binding is followed by the formation of clusters of insulin-receptor complexes. Next, the hormone is apparently internalized by endocytosis [1, 4] and, eventually, it is degraded [5]. It seems likely that the clustering accompanying the formation of the insulin-receptor complexes leads to local disruption of the membrane structure $[1,2]$ and hence is an important factor in the biological activity of insulin in promoting the transport of small molecules, particularly glucose, into the interior of the cell. It has been suggested $[1,2,5]$ that some of insulin's other effects, for example, on protein biosynthesis and down-regulation of receptors, may depend on its internalization.

De Lisi [6] has recently shown theoretically that ligand-induced receptor clustering is capable of producing very large signal amplification in the transduction of information at the cell surface. The situation discussed in his work is directly relevant to that outlined above for the insulin-receptor interaction and the magnitude of the effects which can be generated indicates the need for understanding the details of the process in specific systems. In the present paper I shall propose a model which displays the potential of insulin, in terms of its known molecular properties, for cross-linking receptors into an extensive network following the initial binding of insulin monomer to receptor. The proposed mechanism is shown to account for a wide variety of experimental observations of the relationship between insulin structure and activity, both with respect to comparisons of insulins from different biological species, and of native with chemically modified insulins.

\section{The Model}

A schematic presentation of the possible sequence of events accompanying the binding of insulin monomers to membrane receptors is given in Figure 1. This serves mainly as a framework for discussion; the details should not be taken too literally. The important features are that the receptors are thought to be oligomeric [7] and are free to move in the plane of the membrane [2]. 
For the formation of cross-links it is necessary that the receptors be at least bivalent towards insulin and there is evidence that this is the case $[7,8]$.

The binding of an insulin monomer to a receptor utilizes the insulin groups listed in Table 1 and must generate some biological activity even in the absence of cross-linking (see below). As Pullen et al. [9] have pointed out, these are the groups involved in the contacts between insulin monomers to form the dimer in the 2 zinc porcine insulin crystal plus some additional amino acid residues around the periphery of the monomer-monomer contact region. As documented in Table 1 with data

Table 1. Amino acid residues involved in insulin interactions

\begin{tabular}{|c|c|c|c|c|c|}
\hline \multicolumn{2}{|c|}{ Monomer-monomera } & \multicolumn{2}{|c|}{ Monomer-receptor ${ }^{b}$} & \multicolumn{2}{|c|}{ Dimer-dimer } \\
\hline B8 & Gly & & & B1 & Phe \\
\hline B9 & Ser & & & $\mathrm{B} 2$ & Val \\
\hline B13 & Glu & $\mathrm{B} 22$ & Arg & B13 & Glu \\
\hline B12 & Val & B12 & Val & B4 & Gln \\
\hline B16 & Tyr & B16 & Tyr & B14 & Ala \\
\hline $\mathrm{B} 23$ & Gly & $\mathrm{B} 23$ & Gly & B17 & Leu \\
\hline $\mathrm{B} 24$ & Phe & B24 & Phe & B18 & Val \\
\hline $\mathrm{B} 25$ & Phe & $\mathrm{B} 25$ & Phe & B19 & Cys \\
\hline $\mathrm{B} 26$ & Tyr & B26 & Tyr & $\mathrm{B} 20$ & Gly \\
\hline \multirow[t]{4}{*}{$\mathrm{B} 27$} & Thr & A1 & Gly & A13 & Leu \\
\hline & & A5 & Gln & A14 & Tyr \\
\hline & & A19 & Tyr & A17 & Glu \\
\hline & & A21 & Asn & & \\
\hline
\end{tabular}

a From Blundell et al. [10] (porcine insulin)

b From Pullen et al. [9] (bovine insulin). It is noteworthy that none of the residues involved in the receptor interaction are those required for association of insulin beyond the dimer. from the X-ray analysis of porcine insulin [10], none of the groups making dimer-dimer contacts is involved in the receptor binding. In mammalian insulins which have been studied in solution at neutral $\mathrm{pH}[11,12]$ and in the crystal [10], hydrophobic interactions and hydrogen bonds between these groups are the main forces leading to the post-dimer aggregation. They result in an extended series of polymeric forms in equilibrium in solution, even in the absence of zinc [11]. It is important to note that zinc is not essential for aggregation beyond the dimer in mammalian insulins and also that the effective equilibrium constant for such interaction may be much higher when insulin is bound to a receptor than when it is free in solution [13].

Thus, it is proposed that in those insulins which contain them, the dimer-dimer contact groups (which are still free after the binding of insulin monomers to receptors) associate to form cross-links between receptors in the cell membrane. The process is illustrated in Figure 1 with tetravalent receptors, but only bivalency of receptor and ligand is required for the formation of a two-dimensional network of insulin-receptor complexes of any required extent. The consequent clustering with accompanying disruption of the membrane structure results in enhanced transport of small molecules, such as glucose, into the interior of the cell. Finally, the insulinreceptor complex is apparently internalized [14]; insulin activities which may require its presence and/or that of receptor inside the cell (stimulation of DNA and protein synthesis, regulation of receptor synthesis) are carried out, and eventually the hormone is degraded.

Neither receptor clustering (whether induced by insulin itself or by some other appropriate bi- or multiva-
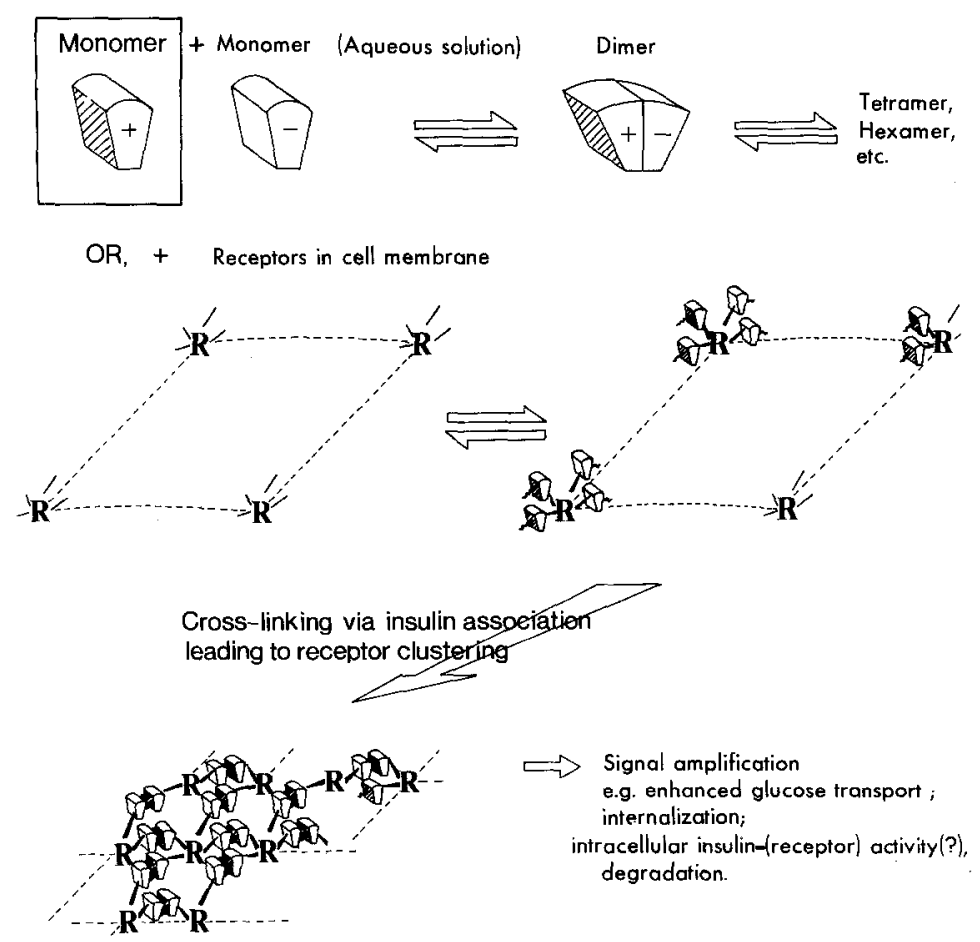

e.g. enhanced glucose transport ; ization degradation
Fig. 1. Schematic diagram of the insulin interactions proposed to account for receptor clustering. In aqueous solution at neutral $\mathrm{pH}$ mammalian insulins self-interact extensively giving rise to a series of polymeric forms in dynamic equilibrium [11, 12]. The monomer is depicted here, in approximation to its crystallographically determined structure [10] as wedgeshaped and the shaded face represents the region containing the side chains which make the dimer-dimer contacts (Table 1). The symbols + and - indicate the upper and lower surfaces of the molecules as viewed down the threefold axis of the hexamer. The insulin receptors in the cell membrane are denoted ' $R$ ' and are shown as tetravalent to insulin monomers though only bivalency is actually required for the formation of a cross-linked network. The lower part of the diagram illustrates the proposal that after binding to the receptor, a process which chiefly utilizes the region involved in dimer formation (Table 1), and which in itself gives rise to some biological activity, the insulin monomers are still capable of self-association via the groups responsible for post-dimer aggregation in solution. The clustering of receptors resulting from the crosslinking thus generated is capable of magnifying greatly responses which depend upon trans-membrane signals [6] 
lent ligand [1-5], nor its part in enhancing insulin-like effects, seem to be seriously in question. What has puzzled some investigators $[1,13]$ is the mechanism whereby insulin could bring such clustering about, since at least bivalency of the binding species is needed and insulin is not an obviously bivalent molecule. The hypothesis advanced in the present paper, based on the known molecular properties of insulin, is that it may be regarded as effectively bivalent through retention of the ability to self-interact, after the formation of a monomer-monomer or monomer-receptor complex, by utilizing inter-molecular contacts which are quite separate from those employed in the former interactions.

The points listed below provide evidence in favour of the idea that the groups utilized in dimer-dimer association in solution are implicated in determining the level of biological potency of insulin. It is shown that this suggested mechanism accounts for a number of experimental observations related to the variability between the activities of different insulins which have not previously found a unifying explanation. The crosslinking of insulin receptors through insulin bridges in the way outlined has implications for the binding response which are also discussed briefly.

\section{Potency of Native Insulins Which do not Associate}

Insulins from non-mammals, for example, hagfish, guinea pig, casiragua and coypu, have low biological activity (as measured, for example, by their ability to facilitate transport of glucose into fat cells) compared with bovine insulin [15]. In fact, bovine insulin is more active in hystricomorph rodents than their own insulins [16]. Significantly, these rodent insulins, unlike bovine insulin, do not self-associate past the dimer stage. Indeed, some of those tested (casiragua, porcupine) do not even form dimers [15]. The theory proposed in this paper makes a direct connection between the two observations. Table 2 documents the amino acid substitutions in the region known to be responsible for dimer-dimer interactions in mammalian insulins [10] for four of the insulins mentioned above. Clearly, these changes would be expected to affect the ability to form aggregates beyond dimers and, in terms of the theory proposed here, to have an equally dramatic effect on receptor clustering and biological potency.

It is characteristic of these low activity insulins that while their biological activities are typically only about $5 \%$ of that of bovine insulin they retain about $25 \%$ of its receptor binding ability [15]. This difference is not unexpected in the light of the present discussion since the operation of two separate sets of interactions, as postulated for maximum potency, implies that the correlation between changes in receptor affinity and potency is not simple. Interestingly $[9,10,16,17]$, the receptor binding region is strongly conserved and one might wonder why the affinity drops even to as low as $25 \%$ of bovine insu-
Table 2. Amino acid substitutions in non-mammalian insulins in the region corresponding to dimer-dimer contacts in porcine insulin

\begin{tabular}{|c|c|c|c|c|c|}
\hline \multicolumn{2}{|c|}{$\begin{array}{l}\text { Porcine } \\
\text { insulin } \\
\text { (from Table 1) }\end{array}$} & \multirow{2}{*}{$\begin{array}{l}\text { Hagfish } \\
{[17]} \\
\text { Arg }\end{array}$} & \multirow{2}{*}{$\begin{array}{l}\text { Casiragua } \\
\text { [16] } \\
\text { Tyr }\end{array}$} & \multirow{2}{*}{$\begin{array}{l}\text { Coypu } \\
{[16]} \\
\text { Tyr }\end{array}$} & \multirow[t]{2}{*}{$\begin{array}{l}\text { Guinea } \\
\text { pig } \\
{[16]}\end{array}$} \\
\hline B1 & Phe & & & & \\
\hline $\mathrm{B} 2$ & Val & Thr & & & \\
\hline B4 & Gln & Gly & & & Arg \\
\hline B13 & Glu & Asn & Asp & Asp & \\
\hline B14 & $\mathrm{Ala}$ & & Thr & Thr & Thr \\
\hline B17 & Leu & Ile & Ser & Ser & Ser \\
\hline B18 & Val & Ala & & & \\
\hline B19 & Cys & & & & \\
\hline $\mathrm{B} 20$ & Gly & & Lys & Arg & Gln \\
\hline A13 & Leu & Ile & Arg & Arg & Arg \\
\hline A14 & Tyr & & Asn & As & $\mathrm{His}$ \\
\hline A17 & Glu & Gln & Leu & Met & Gln \\
\hline
\end{tabular}

lin. Pullen et al. [9] explain this in terms of changes in overall three-dimensional structure associated with other sequence changes in these insulins, even though the binding region itself does not differ in sequence.

It is noted that since some biological activity is present in insulins without the ability to associate, it is not required by the model that cross-linking is required for some transport of metabolites into the cell to occur. Likewise, the ability to dimerize is not necessary for receptor binding to be possible since porcupine insulin, for instance, is active even though it does not form dimers. As the work of Pullen et al. [9] shows, receptor binding is an additive phenomenon and it is not required that all of the monomer-monomer contacts be retained to preserve binding capacity.

A word should perhaps be said about porcupine insulin, which, with only seven amino acid substitutions with respect to bovine insulin, has only $4 \%$ of its activity and $25 \%$ of its binding affinity. It does not dimerize and yet only two of the substitutions are in positions listed in Tables 1 and 2. One is Asp for Arg at B22 (receptor region) and one Gln for Gly at A17 (dimer contact region). However, it seems that the substitution at B22 is a particularly important one [15], thus accounting for the lowering of binding affinity, and it is clear that the other changes of sequence involved, being quite sufficient to prevent association completely, have the power to reduce drastically the biological activity.

Another somewhat atypical hystricomorph insulin is that from the chinchilla. This has $40 \%$ of the receptor binding affinity of bovine insulin and $29 \%$ of its activity [16]. It differs from bovine insulin in sequence at only two positions (B4 and B13), in the dimer-dimer contact region of mammalian insulins and, of the other six substitutions, none occurs at a position listed in Table 1. This would seem to represent a case where loss of receptor affinity is associated with a conformational change rather than the substitutions per se and the larger drop in activity reflects both this effect and the loss of ability to aggregate. 


\section{Modified Insulins}

A great many chemically or enzymatically modified insulins have been prepared, but most of these have been used in studies directed at mapping the receptor binding site $[9,18]$ or may, in retrospect, be seen to have involved residues in that region [10]. Here, a few examples will be presented which seem to bear on the theory under discussion.

The work of Horuk and his colleagues on porcupine insulin has been described above. In that publication [15] the authors also noted that the discrepancy in binding affinity and biological activity had a parallel in modified insulins and cited Sönksen [19] with the comment that they were unable to detect a common factor in these results. The work in question concerned synthetic dimers which had $100 \%$ receptor binding affinity but much lower $(0 \%-60 \%)$ biological activity. A likely reason would appear to be that the linking required to form the synthetic dimers, while not interfering with the receptor binding site, has prevented or hindered further association through the usual dimer-dimer contacts. This explanation provides a simple rationale for the result which reconciles it directly with those obtained with the low potency native insulins.

A similar explanation may apply to the studies with covalent insulin dimers formed by cross-linking with a suberoyl chain to form the dimers B1-B'1 and B1-B'29 [20]. Here again, marked differences between the effect on receptor binding ability (measured in isolated rat adipocytes and rat liver plasma membranes) and biological activity (rates of lipogenesis or inhibition of adrenaline stimulated lipolysis) were noted. The binding retained $92 \%$ and $70 \%$ respectively of its original values but the biological activities were reduced to $38 \%$ and $15 \%$, respectively. Reference to Table 1 shows that residue $\mathrm{B} 1$ is in the dimer-dimer, but not the monomer-receptor, contact region.

Dodson et al. recently studied beef insulin crosslinked at A1 and A29 by diaminosuberic acid (DAS insulin) [21]. The structure of this derivative appeared to be essentially identical with that of native beef insulin as judged from X-ray diffraction data, especially with regard to the conformations of the dimer-forming residues. Moreover, in agreement with this finding, the association constant for dimer formation was the same in the cross-linked as in native beef insulin. However, the biological potency of the cross-linked insulin is reported to be [21] only 5\% that of native. As the authors comment, these results imply that it cannot be the dimerforming residues alone that are responsible for the hormone's normal potency. They suggest that it may be that the monomer needs to adopt a particular conformation before or during binding which the cross-link interferes with. It appears equally possible that some of the reduction in potency (apparently not all, since the binding affinity is also low) could be attributed to alteration of the molecule's ability to aggregate beyond the dimer. The measurements made by Dodson et al. do indeed show a lowering of the association constants for tetramerisation and hexamerisation in the cross-linked insulin.

\section{Nature of the Binding Response}

Scatchard plots of binding results obtained with insulin and receptor preparations are usually in the form identified with negative cooperativity [22]. De Lisi [13] has recently presented theory which shows that receptor clustering can result in Scatchard plots and displacement curves which are indistinguishable from those predicted on the basis of negative cooperativity and heterogeneity. Moreover, the cross-linking model is shown to fit, quantitatively, kinetic data which the other two in their simplest forms cannot.

It seems worth pointing out here that a different approach to cross-linking of either similar [23] or different [24] macromolecules by a ligand has also shown that binding curves are expected to show the characteristics associated with negative cooperativity and, in addition, to be dependent on acceptor concentration. The relevant theory, as with that due to De Lisi, predicts that a maximum biological response can occur in these systems when only a small fraction of the available receptors is occupied, a phenomenon usually referred to as 'spare receptors'. Interestingly, the work of Nichol and his colleagues $[23,24]$ predicts that this maximum in response (or more strictly a maximum in cross-linked complex formation) should occur at a ligand concentration close to the reciprocal of the binding affinity constant for ligand to acceptor. For insulin and its receptor the value measured for this parameter is about $1 \times$ $10^{9} \mathrm{~mol} / 1$ [9], thus $1 / \mathrm{k}=1 \times 10^{-9} \mathrm{~mol} / 1$ about $5 \mathrm{ng} / \mathrm{ml}$, a very reasonable value, as reference to Figure 13 of [5] demonstrates.

\section{Conclusion}

In their paper demonstrating the binding of fluorescently labelled insulin to fibroblastic cells Schlessinger et al. [2] suggested that the aggregation of insulin might be involved in the clustering phenomenon. The discussion presented above indicates clearly that many of the known properties of insulin are consistent with the proposed model. It should, perhaps, be emphasized that this does not depend on details such as the exact number of insulin binding sites per receptor, whether the receptor population is homogeneous, or whether binding curves are truly negatively cooperative. The essence of the proposal is that, after binding to a receptor, some types of insulin are capable of associating further through sites known to interact in solution and in the crystal and that this provides a plausible mechanism for cross-linking receptors and amplifying trans-membrane 
signals. Since the aim of the present work was to suggest a means whereby insulin molecules could cross-link receptors, the discussion has necessarily concentrated on this aspect of the action of insulin. This does not imply that cross-linking operates exclusively to procure insulin's effects. It seems more likely, as De Lisi [13] has stated, that in a real system some mixture of cross-linking, heterogeneity and cooperativity operate.

The present hypothesis could be tested more directly by devising experiments specifically for the purpose. Thus, for example, extensive clustering would not be expected with those insulins which do not associate beyond the dimer. The possibility may also exist for increasing the biological activity of an insulin by modifying it specifically to favour post-dimer aggregation.

\section{References}

1. Kahn CR (1979) What is the molecular basis for the action of insulin? Trends Biochem Sci 4: 263-266

2. Schlessinger J, Schechter Y, Willingham MC, Pastan I (1978) Direct visualization of binding, aggregation and internalization of insulin and epidermal growth factor on living fibroblastic cells. Proc Natl Acad Sci USA 75: 2659-2663

3. Kahn CR, Baird KL, Jarrett DB, Flier JS (1978) Direct demonstration that receptor cross-linking or aggregation is important in insulin action. Proc Natl Acad Sci USA 75: 4209-4213

4. Gorden P, Carpentier J-L, Freychet P, LeCam A, Orci L (1978) Intracellular translocation of Iodine-125-labelled insulin: direct demonstration in isolated hepatocytes. Science 200: 782-785

5. Blecher M (1979) Insulin receptors. In: Vassileva-Popova JG, Jensen EV (eds) Biophysical and biochemical information transfer in recognition. Plenum Press, New York, pp 153-181

6. De Lisi C (1981) The magnitude of signal amplification by ligandinduced receptor clustering. Nature 289: $322-323$

7. Kasuga M, Van Obberghen E, Yamada KM, Harrison LC (1981) Autoantibodies against the insulin receptor recognize the insulin binding subunits of an oligomeric receptor. Diabetes 30:354-357

8. Massague J, Pilch PF, Czech MP (1980) Electrophoretic resolution of three major insulin receptor structures with unique subunit stoichiometries. Proc Natl Acad Sci USA 77: 7137-7141

9. Pullen RA, Lindsay DG, Wood SP, Tickle IJ, Blundell TL, Wollmer A, Krail G, Brandenburg D, Zahn H, Gliemann J, Gammeltoft $S$ (1976) Receptor-binding region of insulin. Nature 259: 369-373

10. Blundell T, Dodson G, Hodgkin D, Mercola D (1972) Insulin: the structure in the crystal and its reflection in chemistry and biology. In: Anfinsen CB Jr, Edsall JT, Richards FM (eds) Advances in protein chemistry 26, Academic Press, New York, pp 279-402

11. Jeffrey PD, Milthorpe BK, Nichol LW (1976) Polymerisation pattern of insulin at pH 7.0. Biochemistry 15: 4660-4665
12. Pekar AH, Frank BH (1972) Conformation of proinsulin. A comparison of insulin and proinsulin association at neutral $\mathrm{pH}$. Biochemistry 11: 4013-4016

13. De Lisi C (1979) Physical chemical and biological implications of receptor clustering. In: De Lisi C, Blumenthal R (eds) Physical and chemical aspects of cell surface events in cellular regulation, Elsevier/North Holland, New York, pp 261-291

14. Desbuquois B, Postel-Vinay M-C (1980) Receptor-mediated internalisation of insulin, glucagon and growth hormone in intact rat liver. A biochemical study. In: Brandenburg D, Wollmer A (eds) Insulin. Chemistry, structure and function of insulin and related hormones. de Gruyter, Berlin, pp 285-292

15. Horuk R, Blundell TL, Lazarus NR, Neville RWJ, Stone D, Wollmer A (1980) A monomeric insulin from the porcupine (Hystrix cristata), an old world hystricomorph. Nature 286: 822-824

16. Horuk R, Goodwin P, O'Connor K, Neville RWJ, Lazarus NR (1979) Evolutionary change in the insulin receptors of hystricomorph rodents. Nature 279:439-440

17. Cutfield JF, Cutfield SM, Dodson EJ, Dodson GG, Emdin SF, Reynolds CD (1979) Structure and biological activity of hagfish insulin. J Mol Biol 132: 85-100

18. De Meyts P, Van Obberghen E, Roth J, Wollmer A, Brandenburg D (1978) Mapping of the residues responsible for the negative cooperativity of the receptor-binding region of insulin. Nature 273: 504-509

19. Sönksen PH (1979) Probing the insulin receptor. Nature 282: 11

20. Willey KP, Tatnell MA, Jones RH, Schüttler A, Brandenburg D (1980) Biological properties of covalent insulin dimers. In: Brandenburg D, Wollmer A (eds) Insulin. Chemistry, structure and function of insulin and related hormones. De Gruyter, Berlin, pp 425-431

21. Dodson GG, Cutfield S, Hoenjet E, Wollmer A, Brandenburg D (1980) Crystal structure, aggregation and biological potency of beef insulin cross-linked at A1 and B29 by diaminosuberic acid. In: Brandenburg D, Wollmer A (eds) Insulin. Chemistry, structure and function of insulin and related hormones. De Gruyter, Berlin, pp 17-27

22. Bar RS, Gorden P, Roth J, Kahn CR, De Meyts P (1976) Fluctuations in the affinity and concentration of insulin receptors on circulating monocytes of obese patients. J Clin Invest 58: 1123-1135

23. Calvert PD, Nichol LW, Sawyer WH (1979) Binding equations for interacting systems comprising multivalent acceptor and bivalent ligand: Application to antigen-antibody systems. J Theor Biol 80: 233-247

24. Nichol LW, Sculley MJ, Jeffrey PD, Teasdale RD (1981) Crosslinking interactions in systems of three reactants: Theory pertaining to their experimental elucidation. J Theor Biol 91: 215-230

Dr. P.D. Jeffrey

Department of Physical Biochemistry

John Curtin School of Medical Research

PO Box 334

Canberra City

ACT 2601

Australia 